\title{
/BOOK REVIEW
}

\section{Probing Diagnostics}

\author{
SANJAY TYAGI
}

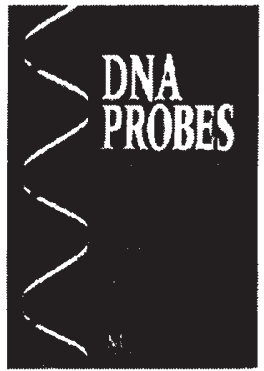

DNA Probes,

George H. Keller and Mark $M$.

Manak, editors, Stockton Press, New York, 1993 , $\$ 90.00$.

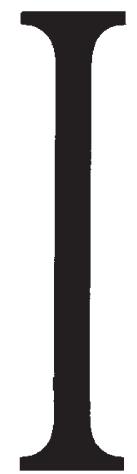

magine that you have a magic reagent to which you add a little droplet of a body fluid from a patient; you wait for a moment and a glow appears in the tube holding the mixture; the glow not only tells you which pathogen is responsible for the patient's illness, it also suggests which drug to use to treat the disease. Also imagine that you can perform this diagnosis even before any symptoms of the disease appear, improving the chances of success with the treatment, and that you can do it for a large population with the same ease.

The creation of such reagents is the promise of nucleic-acid-based diagnostics and is the aspiration of a diverse community of researchers. At the heart of nucleic-acid-based diagnostics is the process of hybridization, in which a strand of a nucleic acid anneals to its complement. Hybridization with nucleic acid probes can be used to determine the identity and pathological potential of an organism with a precision not achievable by conventional antibody or culturebased procedures. DNA Probes, edited by George $\mathrm{H}$. Keller and Mark M. Manak, describes the progress that has recently been made in the implementation of nucleic-acid-based diagnostics. The book outlines the literature with a view toward commercial application of nucleic acid probes. Progress in the field is reflected in the size of the second edition of DNA Probes, which is almost three times the size of the first edition published in 1989.

These diagnostic procedures begin by exposing the target nucleic acids, contacting the probes with the targets, amplifying the probes or the targets, and detecting the products of amplification. Separate sections of DNA Probes are devoted to each of these steps. The set of techniques described for the preparation of samples are conventional and rather labor intensive, which will disappoint those looking for new and easy ways of sample preparation. The section related to amplification strategies conveys the excitement of the field. It contains detailed, illustrated, and clear descriptions of polymerase chain reactions, ligase chain reactions, strand displacement amplifications, transcription-based target amplification, and Qbeta-replicase-mediated amplification of hybridization probes. There are experimental protocols associated with the first four of these techniques, which are

Sanjay Tyagi is an associate at the Public Health Research Institute, 455 First Avenue, New York, NY 10016. (e-mail: sanjay@glycine.phri.nyu.edu.). derived from primary references. A fuller discussion of the performance of these techniques for amplifying nucleic acids in field tests would have been useful. A discussion of hybridization formats and detection methods, which includes conventional, as well as forward-looking techniques, is contained in one section, as are a wide range of hybridization formats. There are clear descriptions of conventional hybridization formats like Southern blotting, as well as of some recently developed formats like reversible target capture, in which the hybrids are immobilized on the surface of paramagnetic particles. Tagging of probes by radioactive, fluorescent, chemiluminescent, and colorogenic labels is described. For those desiring detection of hybridization in homogenous solutions, this section provides the background material. A discussion of how amplification techniques can be combined with detection techniques would have been desirable.

One of the attractive features of nucleic-acid-based diagnostics is that probes can be found very easily for unfamiliar organisms. Using knowledge of the nucleotide sequence of an evolutionarily invariant region of a genome, one can identify probes that characterize a pathogenic species or even a strain. There is only a fragmentary discussion of the procedures that have been devised for the identification of probes for detecting organisms. However, a section written by Rohan G. Kroll on the microbiological analysis of foods contains large lists of probes identified for the detection of food-borne organisms.

The history and theory of hybridization is discussed briefly in the first section and will be a good guide for those who are newly recruited into the nucleic-acidbased assay business. What makes nucleic-acid-based diagnostics possible is the solid-phase synthesis of DNA and molecular cloning techniques. DNA Probes includes two large sections on these subjects, which do not really belong in this volume as there are already excellent references in the field concerning them.

The application of nucleic acid probes is not limited to the diagnosis of infectious diseases. These probes will find applications in the detection of genetic disorders and cancer, the detection of biological pollutants, the analysis of soil, and in food testing and forensic analysis. DNA Probes contains specialized chapters on each of these topics. Although penned by different authors, an effort has been made to coordinate style and content.

DNA Probes will be a useful reference for those interested in reducing to practice the idea of diagnosis by nucleic acid probes. 